\title{
Prediction of Factors Influencing the Timing and Prognosis of Early Tracheostomy in Patients with Multiple Rib Fractures using Propensity Score Matching Analysis
}

\section{Bing Zhang}

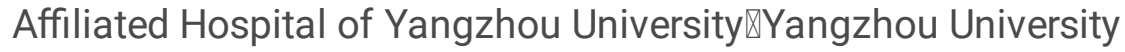

\section{GongKe Li}

Affiliated Hospital of Yangzhou University,Yangzhou University

\section{YuRong Wang}

Affiliated Hospital of Yangzhou University,Yangzhou University

yong li ( $\nabla$ yzliyong0304@163.com )

The Affiliated Hospital of Yangzhou University https://orcid.org/0000-0001-9256-6690

\section{Original research}

Keywords: early tracheostomy, multiple rib fractures, propensity score matching

Posted Date: November 10th, 2021

DOI: https://doi.org/10.21203/rs.3.rs-1026962/v1

License: (1) This work is licensed under a Creative Commons Attribution 4.0 International License. Read Full License 


\section{Abstract}

Objective To explore the factors affecting the timing and prognosis of early tracheostomy(within 7 days after tracheal intubation) in patients with multiple rib fractures.

Methods A retrospective analysis of the medical history of 222 patients with multiple rib fractures who were admitted to the department of emergency intensive care unit(EICU) of the affiliated hospital of Yangzhou University from February 2015 to October 2019 underwent early tracheostomy. According to the time from tracheal intubation to tracheostomy after admission, the patients were divided into two groups: early tracheostomy group (within 7 days after tracheal intubation,ET) and late tracheostomy group (after the 7th day, LT). The propensity score matching analysis technique was used to compare the differences between the two groups in a 1:1 ratio.

Results A total of 222 patients were enrolled, with 118 in the ET group and 104 in the LT group. After matching, 87 in the ET group and 87 in the LT group. The proportion of acute respiratory distress syndrome(ARDS)[59(67.8\%)], the volume of pulmonary contusion (VPC)[33.8 11.4], and number of total rib fractures (NTRF) $[10.8 \pm 2.7]$ in the ET were significantly higher than those in the LT group, $P<0.05$. Binary Logistic regression analysis showed that ARDS[OR=3.740, 95\% Cl(1.441, 9.711)], VPC[OR=1.087, 95\% $\mathrm{Cl}(1.052$, 1.124)], and NTRF $[O R=1.775,95 \% \mathrm{Cl}(1.439,2.188)]$ were independent risk factors for ET.The Pearson analysis showed that VPC and NTRF had significant correlation $(R=0.369, P=0.01)$, ARDS and VPC had low degree correlation $(R=0.179, P=0.018)$, while ARDS and NTRF had no significant correlation $(R=0.132, P=0.110)$. Receiver operating characteristic $(\mathrm{ROC})$ curve analysis showed that the area under VPC and NTRF curves [0.832(95\% Cl: $0.770 ~ 0.893), 0.804(95 \% \mathrm{Cl}: 0.740 \sim 0.868)]$ were significantly more than the number of rib fractures(NFR), glasgow coma scale(GCS), and injury severity score(ISS), $P<0.05$. COX regression analysis showed that patients with underwent ET survived significantly better than the $L T, P<0.05$.

Conclusions We found that ARDS, VPC, and NTFR were independent risk factors for ET; VPC $\geq 23.9 \%$ and (or) NTRF $\geq 8.5$ could be used as predictors of ET in patients with multiple rib fractures. There was a linear relationship between NTRF and VPC. ET might benefit patients with multiple rib fractures.

\section{Introduction}

Multiple rib fractures(the number of fractured ribs is more than 3 ) are usually caused by chest trauma, which might be combined with pulmonary contusion, hemothorax, pneumothorax, and possibly life-threatening lung injury ${ }^{[1]}$. Patients with multiple rib fractures and pneumonia might require tracheostomy. It was reported that incidence of tracheostomy in critically injured patients with acute respiratory failure was up to $59.0 \%$, with an average of $19.6 \%{ }^{[2]}$.

ET may benefit patients with multiple rib fractures. These benefits include shortening the time of sedation ${ }^{[3]}$, reducing the time of ventilator use, shortening the ICU lenght of stay(ICULOS) and hospital lenght of stay(HLOS) ${ }^{[4]}$, and even reducing the incidence of pneumonia and short-term mortality ${ }^{[3-6]}$. However, tracheostomy can also cause complications such as bleeding, tracheal stenosis, incision skin ulcers ${ }^{[7,8]}$. Therefore, how to predict the timing and prognosis of ET is a difficult problem for clinicians. However, there 
were very few relevant studies at present, only single-center and retrospective studies. It has been reported that severe GCS score $(\leq 8)$, flail chest, and severe trauma score were risk factors that might cause patients with multiple rib fractures to prolong the time of mechanical ventilation ${ }^{[9]}$. Some researchers had also found that the clinical application of tracheostomy predictive models were limited because of poor predictive sensitivity and the positive predictive value of only $30-45 \%{ }^{[10]}$.

Therefore, we used propensity score matching analysis to verify the general data and clinical results of patients with multiple rib fractures. Our purpose was to find suitable indicators to predict the timing and prognosis of early tracheostomy in patients with multiple rib fractures.

\section{Patients And Methods 1.1 Research object}

This was a retrospective cohort study. Our study subjects were patients with multiple rib fractures who underwent tracheostomy and were radiologically confirmed (computed tomography scans,CTs) in the ICU of the affiliated hospital of Yangzhou University between February 2015 and October 2019. Other data were extracted from the inpatient registries and patient electronic medical records. Our inclusion criteria: age $>18$ years; multiple rib fractures diagnosed by computer tomography; due to chest injury, patients underwent tracheostomy treatment after admission. We excluded patients who were $<18$ years old, who were cardiac arrest, and who were underwent tracheostomy due to severe traumatic brain injury, burn, and spine injury. According to the 2009 tracheostomy timing management guide ${ }^{[1]}$, the patients were divided into two groups: ET was defined as tracheostomy within 7 days after tracheal intubation, and LT was after the 7 th day.

\subsection{Data collection}

We collectted variables included age, gender, glasgow coma scale(GCS), injury severity score (ISS), complications(cardiovascular disease and lung disease), traumatic brain injury(TBI), acute respiratory distress syndrome(ARDS), volume of pulmonary contusion(VPC), number of ribs fractured, number of total fractures of the ribs(NTRF), first rib fracture, flail chest, sternum fractures, spine coinjuries, maxillofacial coinjuries, initial value of blood lactate, hemothorax, pneumothorax, timing of tracheostomy, duration of mechanical ventilation, duration of tracheostomy, hospital length of stay (HLOS), ICU length of stay(ICULOS), thoracic close drainage, number of fiberbronchoscope, multidrug resistance bacteria, ventilator associated pneumonia, antibiotic use day, sedatives and analgesics use day, and 28-day mortality. Each slice of the admission spiral chest CTs was documented and reconstructed in 3D by Advantage Workstation 4.3 computer software (GE Healthcare, Waukesha, WI), so that precise contusion volumes could be measured. Bilateral pulmonary fields were also reconstructed and pulmonary volume was measured. Total PC volume for both pulmonary fields was expressed as a percentage of total pulmonary volume. We defined ARDS according to the Berlin definition ${ }^{[12]}$.

\subsection{Treatment method}


All patients received standard treatments: analgesia and sedation, bronchoscopy and alveolar lavage, and chest physical therapy. (1) Analgesia and sedation are all systemic drugs, our purpose is to enable patients to breathe effectively, promote airway secretion clearance and reduce the formation of atelectasis; (2) Bronchoscopy and alveolar lavage It can further optimize the removal of patients' airway secretions and the prevention of pneumonia; (3) Chest physical therapy includes sputum suction, artificial airway management (turning over and buckling the back to promote sputum drainage), mechanical sputum assisted removal of airway secretions, chest belt external fixation, closed thoracic drainage, nasal or oral endotracheal intubation to establish artificial positive airway pressure and tracheostomy treatment. Tracheostomy was performed according to classic indications ${ }^{[13]}$. All operations were performed by physicians with extensive clinical experience. The specific methods were carried out in accordance with the latest guidelines ${ }^{[14]}$.

\subsection{Statistical analysis}

IBM SPSS software version 22.0 (IBM, Armonk, NY) for statistical analysis, continuous variables and normal distribution data were represented by mean \pm standard deviation, non-normal distribution was represented by median (inter quartile range), and categorical variable data was represented by percentage (rate). The control coh ort of early and late tracheostomy patients obtained by propensity score matching method, the matching ratio is $1: 1$, the caliper value is 0.2 , and age, ISS and tracheostomy time are used as covariates without replacement. The logistic regression was used to predict the independent risk factors of early tracheostomy, and Pearson correlation analysis was performed on the independent factors generated. ROC curve compared the significance of the risk factors affecting early tracheostomy. Kaplan-Meier and COX survival analysis were used to analyze the influencing factors of patients' 28-day survival.

\section{Results}

\subsection{Characteristics of patients}

A total of 251 patients with tracheostomy were involved, of which 29 were excluded [ 8 cases age $<18$ years old, 5 cases with cardiac arrest, and tracheostomy due to severe traumatic brain injury(9 cases), burn( 2 case), and ( 5 cases)], therefore, 222 patients finally met the enrollment criteria.The average age is 50 years old, 160 males $(72.1 \%)$. The most common injury was traffic accident injuries (156 cases, $70.3 \%)$, followed by high fall injuries (41 cases, $18.5 \%)$, fall injuries (12 cases, $5.4 \%$ ), and crush injuries (11 cases, $4.9 \%)$ ), unexplained injury ( 2 cases, $0.9 \%$ ). Among patients who underwent tracheostomy within 7 days after intubation, 118 cases (53.2\%) met the ET, and 104 cases (46.8\%) were included in the LT. On account of propensity score matching analysis, There were 87 patients in the ET group and LT group respectively. Hence, a total of 174 patients were sampled for result analysis (Figure 1).

Before propensity score matching, significantly difference in cardiovascular disease, ARDS, VPC, NTRF, and hemothorax between the ET and the LT. After propensity score matching, ARDS, VPC, and NTRF were significantly different; Nevertheless,Age, male, GCS, ISS , lung disease, TBI , number of fractured ribs, first rib fracture, combined injury (flail chest, maxillofacial, spine, hemothorax, pneumothorax) and initial value of 
blood lactate were not significantly different; In addition, Timing of tracheostomy for patients in ET and LT

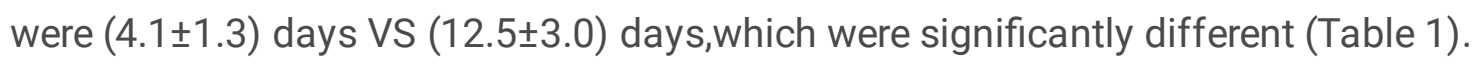

\subsection{Outcome of patients}

Before propensity score matching, the ratio of closed thoracic drainage and fungal infection in the ET was higher than that of the LT, while no significant difference between after matching. the 28-day mortality of the ET group was lower than that of the LT, and there was a statistical difference (Table 2).

The results of binary logistic regression analysis of multiple factors showed that ARDS, VPC and NTRF were three independent risk factors for patients with multiple rib fractures who underwent early tracheostomy (Figure 2). Pearson correlation analysis of three independent factors showed that: VPC and NTRF are moderately related, $R=0.369, P=0.001$; ARDS and VPC are lowly related, $R=0.179, P=0.018$; NTRF and ARDS are not obviously correlation, $\mathrm{R}=0.132, P=0.110$ (Figure 3 ).

ROC curve analysis showed that the area under the curve of VPC and NTFR were 0.804 and 0.832 , respectively, $P=0.001$. While that of GCS, ISS and NFR had no significant difference, $P$-value $₫ 0.05$ (Figure 4 ). We calculated the maximum value of the VPC's Youden index [sensitivity - (1-specificity)] is 0.633 ,corresponding sensitivity $=0.828$. SPSS statistical software could calculate the corresponding value of VPC is 23.9.As well,we ccould also calculate that the maximum value of the NTFR's Youden index is 0.474 , corresponding sensitivity=0.839. SPSS statistical software could also calculate the corresponding value of NTFR is 8.5 .

Kaplan-Meier survival analysis showed that the 28-day survival of ET was significantly better than that of $\mathrm{LT}, P=0.01$ (Figure 5). In addition, COX survival analysis showed that the timing of tracheostomy $(H R=2.51$ $95 \% C / 1.12-5.57, P=0.004)$ and age $(H R=1.5395 \% C / 1.00-2.05, P=0.042)$ of patients had a significant impact on the 28-day survival of patients with multiple rib fractures. 
Table 1

Comparison of baseline data of patients before and after propensity score matching

Variables

Before propensity matching

After propensity matching

\begin{tabular}{|c|c|c|c|c|c|c|}
\hline & $\begin{array}{l}\mathrm{ET} \\
\bigotimes \mathrm{N}=118 \rrbracket\end{array}$ & $\begin{array}{l}\mathrm{LT} \\
\square \mathrm{N}=104 \rrbracket\end{array}$ & $\begin{array}{l}\text { P- } \\
\text { value }\end{array}$ & $\mathrm{ET} \otimes \mathrm{N}=87 \rrbracket$ & $\begin{array}{l}\mathrm{LT} \\
\bigotimes \mathrm{N}=87 \rrbracket\end{array}$ & $\begin{array}{l}\mathrm{P} \text { - } \\
\text { value }\end{array}$ \\
\hline Age, years, points & $50.5 \pm 15.6$ & $47.5 \pm 18.2$ & 0.196 & $48.2 \pm 16.1$ & $47.6 \pm 20$ & 0.818 \\
\hline Male sex, n (\%) & $84 \otimes 71.1 \rrbracket$ & $76 \otimes 73.1 \rrbracket$ & 0.754 & $67 \otimes 77.0 \otimes$ & $\begin{array}{l}59 \\
\square 67.8 \rrbracket\end{array}$ & 0.175 \\
\hline GCS at admission, points & $9.1 \pm 4.3$ & $9.0 \pm 4.5$ & 0.893 & $9.2 \pm 4.3$ & $8.9 \pm 4.6$ & 0.660 \\
\hline ISS, points & $38.9 \pm 7.2$ & $40.1 \pm 9.4$ & 0.294 & $39.3 \pm 7.8$ & $40.0 \pm 8.8$ & 0.580 \\
\hline Cardiovascular diseases, n (\%) & $21 \rrbracket 17.8 \rrbracket$ & $8 \otimes 7.7 \rrbracket$ & 0.026 & 13凶14.9凶 & $8 \rrbracket 9.2 \rrbracket$ & 0.245 \\
\hline Lung disease, n (\%) & $12 \rrbracket 10.2 \rrbracket$ & $12 \rrbracket 11.5 \rrbracket$ & 0.743 & $9 \rrbracket 10.3 \rrbracket$ & 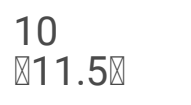 & 0.808 \\
\hline Traumatic Brain Injury, n (\%) & $84 \otimes 71.2 \rrbracket$ & $72 \rrbracket 69.2 \rrbracket$ & 0.750 & $60 \rrbracket 69.0 \rrbracket$ & $\begin{array}{l}62 \\
\square 71.3 \rrbracket\end{array}$ & 0.740 \\
\hline $\begin{array}{l}\text { Acute Respiratory Distress } \\
\text { Syndrome, } \mathrm{n}(\%)\end{array}$ & $76(64.4)$ & $48(46.2)$ & 0.006 & $59 \llbracket 67.8 \rrbracket$ & $\begin{array}{l}37 \\
\otimes 42.5 \rrbracket\end{array}$ & 0.001 \\
\hline $\begin{array}{l}\text { Volume of pulmonary contusion, } \\
\text { points }\end{array}$ & $21.1 \pm 18.0$ & $5.9 \pm 8.7$ & 0.000 & $33.8 \pm 11.4$ & $20.1 \pm 9.8$ & 0.000 \\
\hline Number of ribs fractured, points & $5.8 \pm 2.1$ & $6.1 \pm 2.1$ & 0.218 & $5.7 \pm 2.1$ & $6.2 \pm 2.1$ & 0.167 \\
\hline Number of total rib fractures, points & $9.1 \pm 2.4$ & $7.9 \pm 2.2$ & 0.001 & $10.8 \pm 2.7$ & $7.9 \pm 2.0$ & 0.001 \\
\hline First rib fractures, n (\%) & $40(33.9)$ & $34(32.7)$ & 0.849 & $30 \varangle 34.5 \rrbracket$ & $\begin{array}{l}29 \\
\rrbracket 33.3 \rrbracket\end{array}$ & 0.873 \\
\hline Sternum fractures, $\mathrm{n}(\%)$ & $37(31.4)$ & $31(29.8)$ & 0.803 & $26 \varangle 29.9 \rrbracket$ & 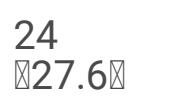 & 0.738 \\
\hline Flail chest, $\mathrm{n}(\%)$ & $20(16.9)$ & $24(23.1)$ & 0.253 & 14ه16.1ه & $\begin{array}{l}22 \\
\otimes 25.3 \otimes\end{array}$ & 0.134 \\
\hline Spine coinjuries, $\mathrm{n}(\%)$ & $12 \bowtie 10.2 \rrbracket$ & $4 \llbracket 3.8 \rrbracket$ & 0.069 & $10 \otimes 11.5 \rrbracket$ & $4 \llbracket 4.6 \rrbracket$ & 0.094 \\
\hline Maxillofacial coinjuries, $\mathrm{n}(\%)$ & $8 \rrbracket 6.9 \rrbracket$ & $12 \otimes 11.5 \rrbracket$ & 0.232 & $8 \rrbracket 9.4 \rrbracket$ & 9囚10.3】 & 0.838 \\
\hline initial value of blood lactate, points & $4.0 \pm 2.9$ & $4.1 \pm 2.2$ & 0.836 & $4.1 \pm 2.9$ & $3.8 \pm 2.1$ & 0.509 \\
\hline Hemothorax, n (\%) & $58 \rrbracket 49.2 \rrbracket$ & $32 \bowtie 32.0 \rrbracket$ & 0.010 & 41区47.1区 & 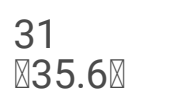 & 0.124 \\
\hline Pneumothorax, n (\%) & $70 \rrbracket 59.3 \rrbracket$ & $56 \rrbracket 53.8 \rrbracket$ & 0.411 & $50 \otimes 57.5 \rrbracket$ & $\begin{array}{l}51 \\
\square 58.6 \rrbracket\end{array}$ & 0.878 \\
\hline $\begin{array}{l}\text { Timing of tracheostomy, day, } \\
\text { points }\end{array}$ & $4.0 \pm 1.3$ & $12.6 \pm 3.1$ & 0.000 & $4.1 \pm 1.3$ & $12.5 \pm 3.0$ & 0.001 \\
\hline
\end{tabular}

ET, early tracheostomy; LT, late tracheostomy; GCS, glasgow coma scale; ISS, injury severity score. 


\section{Table 2}

Comparison of clinical outcomes of patients before and after propensity score matching

\begin{tabular}{|c|c|c|c|c|c|c|}
\hline \multirow[t]{2}{*}{ Outcomes } & \multicolumn{3}{|c|}{ Before propensity matching } & \multicolumn{3}{|c|}{ After propensity matching } \\
\hline & $\begin{array}{l}E T \\
\bigotimes N=118 \rrbracket\end{array}$ & $\begin{array}{l}\mathrm{LT} \\
\bigotimes N=104 \rrbracket\end{array}$ & $\begin{array}{l}\mathrm{P}- \\
\text { value }\end{array}$ & $\begin{array}{l}E T \\
\bigotimes N=87 \rrbracket\end{array}$ & $\begin{array}{l}\mathrm{LT} \\
\bigotimes \mathrm{N}=87 \rrbracket\end{array}$ & $\begin{array}{l}\mathrm{P}- \\
\text { value }\end{array}$ \\
\hline $\begin{array}{l}\text { Duration Of mechanical ventilation, } \\
\text { days }\end{array}$ & $13.5 \pm 4.1$ & $15.7 \pm 5.5$ & 0.078 & $13.9 \pm 3.0$ & $15.9 \pm 4.7$ & 0.190 \\
\hline Duration of tracheostomy, days & $27.3 \pm 6.5$ & $35.8 \pm 8.2$ & 0.180 & $28.4 \pm 6.1$ & $39.4 \pm 7.1$ & 0.143 \\
\hline Hospital lenght of stay, days & $18.3 \pm 6.0$ & $17.7 \pm 5.1$ & 0.855 & $19.3 \pm 6.6$ & $17.0 \pm 5.8$ & 0.521 \\
\hline ICU lenght of stay, days & $7.1 \pm 2.7$ & $11.5 \pm 3.6$ & 0.145 & $8.7 \pm 2.1$ & $11.6 \pm 2.7$ & 0.412 \\
\hline Thoracic close drainage, $\mathrm{n}(\%)$ & $66(55.9)$ & $44(42.3)$ & 0.043 & $46(52.9)$ & $39(44.8)$ & 0.288 \\
\hline $\begin{array}{l}\text { Number of fiber bronchoscope use, } \\
\text { points }\end{array}$ & $2.5 \pm 5.4$ & $1.7 \pm 2.9$ & 0.194 & $2.8 \pm 5.9$ & $1.7 \pm 2.8$ & 0.129 \\
\hline Multidrug resistance bacteria, $\mathrm{n}(\%)$ & $36(30.5)$ & $34(32.6)$ & 0.335 & $29(33.3)$ & $28(32.2)$ & 0.732 \\
\hline $\begin{array}{l}\text { Ventilator associated pneumonia, } \mathrm{n} \\
(\%)\end{array}$ & $24(20.3)$ & 20(19.2) & 0.657 & 18(20.7) & 19(21.8) & 0.732 \\
\hline Antibiotic use, days & $10.0 \pm 3.4$ & $9.2 \pm 2.6$ & 0.722 & $13.5 \pm 4.3$ & $14.1 \pm 4.6$ & 0.876 \\
\hline Sedatives and analgesics use, days & $11.4 \pm 9.1$ & $13.7 \pm 8.6$ & 0.060 & $11.6 \pm 9.2$ & $13.5 \pm 8.3$ & 0.151 \\
\hline 28-day mortality, n (\%) & $14(11.9)$ & $29(27.9)$ & 0.003 & $9(10.3)$ & $28(32.2)$ & 0.001 \\
\hline
\end{tabular}

\section{Discussion}

Many researchers tried to predict the influencing factors of early tracheostomy in ICU patients. Most of these studies focused on specific subgroups, such as patients with multiple injuries (cranial trauma, spinal injury, burns) ${ }^{[15-18]}$, spontaneous cerebral hemorrhage ${ }^{[19-20]}$, and hypoxic/hypoxic-ischemic encephalopathy ${ }^{[21]}$. However, there was a lack of large-scale observational studies on patients with chest trauma, especially multiple rib fractures.

Three key findings were drawn from this study. First, ARDS, VPC, and NTFR were independent risk factors for ET; Secondly, VPC $\geq 23.9 \%$ and (or) NTRF $\geq 8.5$ could be used as predictors of ET in patients with multiple rib fractures. In addition, there was a significant correlation between VPC and NTFR. With the increase of NTFR, VPC also tended to increase significantly. Finally, we found that ET might benefit patients with multiple rib fractures. 
Several retrospective studies had shown that severe brain injuries, flail chest, severe thoracic trauma score, lung contusion, and rib fractures ${ }^{[22-23]}$ were the risk factors of multiple rib fractures patients with mechanical ventilation for more than 7 days. Nevertheless,our study excluded patients whose coma was longer than 72 hours due to TBI, in order to reduce the impact of tracheostomy for prolonged coma. In addition, we counted the NTFR in each enrolled patient, which quantified the severity of chest injury more than flail chest. Fokin et al $^{[24]}$ found that no matter the total number of rib fractures $\geq 5$ or $\geq 6$, the timing of tracheostomy was not affected. However, our study found that when the NTFR $\geq 8.5$, patients might require early tracheostomy, and benefit patients with multiple rib fractures. We believe that this may be the threshold of the number of rib fractures affecting the outcome of the patient. The studies of Battle et al ${ }^{[25]}$ and Shulzhenko et al ${ }^{[26]}$ also showed the same results. In addition, previous studies found that VPC could quantify the severity of lung contusion ${ }^{[11,27-28]}$. For the first time, we used VPC as a research variable for patients with multiple rib fractures underwent tracheostomy, and reached a conclusion. When VPC $\geq 23.9$, it indicated that the patient might need early tracheostomy, and it was also related to the patient's survival benefit. The studies of Mahmood et al [27] and Wang et $\mathrm{al}^{[28]}$ were consistent with ours. Finally, we found that there was a significant correlation between NTFR and VPC. Trinkled et al ${ }^{[29]}$ also confirmed that flail chest-related respiratory insufficiency was caused by a potential lung contusion, and that the shear force generated by the fracture caused lung tissue and blood vessel damage ${ }^{[30]}$.

Several studies had shown that surgical internal fixation significantly reduced HLOS , ICU LOS, and the incidence of pneumonia ${ }^{[31-32]}$. However, our study showed that there were no significant differences between in the two groups. The population included in our study was patients with severe chest trauma who received mechanical ventilation. Such patients often had longer hospital stay and high incidence of ventilatorassociated pneumonia. Our study foud that the 28-day mortality of patients in ET group was lower than that in LT group, and the results of survival analysis also verify that the 28-day survival of patients in ET was better than that in LT. This indicated that early tracheostomy also was related to the patient's survival benefit, which was consistent with the view of Raimondi et al ${ }^{[33]}$. However, Fokin et al ${ }^{[23]}$ and Kang et al ${ }^{[34]}$ reported that early tracheostomy did not reduce the mortality of trauma patients. The reason was that they found that mortality was related to traumatic brain injury and GCS was less than 8 on admission, and most deaths occurred in 3 to 5 weeks after admission. We believed that the cause of the difference in mortality might be due to selection bias or different monitoring modes. In addition, we believed that if the patients were found to be a high-risk group of tracheostomy, ET might benefit them.Finally, we emphasized the individualized treatment plan, and should not choose ET in order to reduce the mortality.

However, our report had several limitations. First of all, it was a retrospective and observational study, so subject to the limitations of this type of research and only involves data from one city in Country. But we believed that our study contained a large amount of data for all this group, so it was a good representation of the characteristics of this type of trauma patients. In addition, we speculated that there may be other undiscovered indicators. In the future, it is an urgent need to establish a more complete prediction model to predict the timing and prognostic of early tracheostomy in patients with multiple rib fractures.

\section{Conclusion}


We found that ARDS, VPC, and NTFR were independent risk factors for ET; VPC $\geq 23.9 \%$ and (or) NTRF $\geq 8.5$ could be used as predictors of ET in patients with multiple rib fractures. There was a linear relationship between NTRF and VPC. ET might benefit patients with multiple rib fractures.

\section{Abbreviations}

EICU, emergency intensive care unit; ET, early tracheostomy;LT, late tracheostomy;ARDS, acute respiratory distress syndrome;VPC, volume of pulmonary contusion; NTRF, number of total rib fractures; ROC, receiver operating characteristic; NFR, number of rib fractures; GCS, glasgow coma scale; ISS,injury severity score;ICULOS, intensive care unit lenght of stay; HLOS, hospital lenght of stay; CTs, computed tomography scans; $\mathrm{TBI}$, traumatic brain injury; $\mathrm{HR}$, hazard ratio; $95 \% \mathrm{Cl}, 95 \%$ confidence interval.

\section{Declarations}

\section{ETHICS APPROVAL AND CONSENT TO PARTICIPATE}

Our study was discussed and approved by the Ethics Committee of the Affiliated Hospital of Yangzhou University. Ethical Review Opinion No.2020-YKL12-23-(01).

\section{CONSENT FOR PUBLICATION}

Any individual person's data in our study(including individual details and images) had been obtained from that person.

\section{AVAILABILITY OF DATA AND MATERIALS}

Please contact author for data requests.

\section{COMPETING INTERESTS}

The authors declare that they have no competing interests.

\section{FUNDING}

This study was supported by Science and technology innovation cultivation fund of Yangzhou University(2019CXJ208) and Jiangsu Province 333 High-level Talent Training Project(BRA2020176).

\section{AUTHORS' CONTRIBUTIONS}

GL, and YW contributed to determining variables, extracting variables from the trauma registry, providing input, and finally approving the manuscript. Besides ,YL was a contributor to the research design, responsible 


\section{ACKNOWLEDGEMENTS}

Not applicable.

\section{References}

1. Nummela MT, Bensch FV, Pyhältö TT, Koskinen SK. Incidence and Imaging Findings of Costal Cartilage Fractures in Patients with Blunt Chest Trauma: A Retrospective Review of 1461 Consecutive Whole-Body CT Examinations for Trauma. Radiology. 2018 Feb;286(2):696-704.

2. Nathens $A B$, Rivara FP, Mack $C D$, et al. Variations in rates of tracheostomy in the critically ill trauma patient. Crit Care Med. 2006 Dec;34(12):2919-24.

3. Meng L, Wang C, Li J, Zhang J. Early vs late tracheostomy in critically ill patients: a systematic review and meta-analysis. Clin Respir J 2016;10:684-692.

4. Mubashir T, Arif AA, Ernest P, et al. Early Versus Late Tracheostomy in Patients With Acute Traumatic Spinal Cord Injury: A Systematic Review and Meta-analysis. Anesth Analg. 2020 Sep 30.

5. Rumbak MJ, Newton M, Truncale T, et al. A prospective, randomized, study comparing early percutaneous dilational tracheotomy to prolonged translaryngeal intubation (delayed tracheotomy) in critically ill medical patients. Crit Care Med 2004;32:1689-94.

6. Mallick A, Bodenham AR. Tracheostomy in critically ill patients. Eur J Anaesthesiol. 2010;27:676-682.

7. Terragni PP, Antonelli M, Fumagalli R, et al. Early vs late tracheotomy for prevention of pneumonia in mechanically ventilated adult ICU patients: a randomized controlled trial. JAMA. 2010;303(15):14831489.

8. Young D, Harrison DA, Cuthbertson BH, Rowan K; for the TracMan collaborators. Effect of early vs late tracheostomy placement on survival in patients receiving mechanical ventilation: the TracMan randomized trial. JAMA. 2013;309(20):2121-2129.

9. Okabe Y. Risk factors for prolonged mechanical ventilation in patients with severe multiple injuries and blunt chest trauma: a single center retrospective case-control study. Acute Med Surg. 2018 Jan 31;5(2):166-172.

10. Casamento A, Bailey M, Robbins R, et al.Patient characteristics, incidence, technique, outcomes and early prediction of tracheostomy in the state of Victoria, Australia. J Crit Care. 2018 Apr;44:278-284.

11. Holevar M,Dunham JC,Brautigan R,et al. Practice management guidelines for timing of tracheostomy: the EAST Practice Management Guidelines Work Group [J]. J Trauma, 2009, 67(4):870-874.

12. ARDS Definition Task Force, Ranieri VM, Rubenfeld GD, Thompson BT, et al. Acute respiratory distress syndrome: the Berlin Definition. JAMA. 2012 Jun 20;307(23):2526-33.

13. Rosero EB, Corbett J, Mau T, Joshi GP. Intraoperative Airway Management Considerations for Adult Patients Presenting With Tracheostomy: A Narrative Review. Anesth Analg. 2021 Apr 1;132(4):1003-1011. 
14. Trouillet JL, Collange O, Belafia F, et al.Tracheotomy in the intensive care unit: guidelines from a French expert panel.Ann Intensive Care. 2018 Mar 15;8(1):37.

15. Branco BC, Plurad D, Green DJ, et al. Incidence and clinical predictors for tracheostomy after cervical spinal cord injury: a national trauma databank review. J Trauma 2011;70:111-5.

16. Trouillet JL, Collange O, Belafia F, et al.Tracheotomy in the intensive care unit: guidelines from a French expert panel.Ann Intensive Care. 2018 Mar 15;8(1):37.

17. Robba C, Galimberti S, Graziano F, et al.Tracheostomy practice and timing in traumatic brain-injured patients: a CENTER-TBI study. Intensive Care Med. 2020 May; 46(5): 983-994.

18. Tsuchiya A, Yamana $H$, Kawahara $T$, et al. Tracheostomy and mortality in patients with severe burns: $A$ nationwide observational study. Burns. 2018 Dec;44(8):1954-1961.

19. Huttner HB, Kohrmann M, Berger C, et al. Predictive factors for tracheostomy in neurocritical care patients with spontaneous supratentorial haemorrhage. Cerebrovasc Dis 2006;21:159-65.

20. Szeder V, Ortega-Gutierrez S, Ziai W, Torbey MT. The TRACH score: clinical and radiological predictors of tracheostomy in supratentorial spontaneous intracerebral hemorrhage. Neurocrit Care 2010;13:40-6.

21. Allareddy V, Rampa S, Nalliah RP, et al. Prevalence and predictors of gastrostomy tube and tracheostomy placement in anoxic/hypoxic ischemic encephalopathic survivors of in-hospital cardiopulmonary resuscitation in the United States. PLoS One 2015;10:e0132612.

22. Dehghan N, de Mestral C, McKee MD, et al. Flail chest injuries: a review of outcomes and treatment practices from the National Trauma Data Bank. J. Trauma Acute Care Surg. 2014; 76: 462-8.

23. Bruce S, James E, Faran B, et al. Management of pulmonary contusion and fflail chest: an Eastern Association for the Surgery of Trauma practice management guideline. J. Trauma Acute Care Surg. 2012; 73 (Suppl 4): 351-61.

24. Fokin AA, Wycech J, Chin Shue K, et al. Early Versus Late Tracheostomy in Trauma Patients With Rib Fractures. J Surg Res. 2020 Jan;245:72-80.

25. Battle CE, Hutchings $\mathrm{H}$, Evans PA. Risk factors that predict mortality in patients with blunt chest wall trauma: a systematic review and meta-analysis. Injury. 2012;43:8-17.

26. Shulzhenko NO, Zens TJ, Beems MV, et al. Number of rib fractures thresholds independently predict worse outcomes in older patients with blunt trauma. Surgery. 2017;161:1083-1089.

27. Mahmood I, El-Menyar A, Younis B, et al. Clinical Significance and Prognostic Implications of Quantifying Pulmonary Contusion Volume in Patients with Blunt Chest Trauma. Med Sci Monit. 2017 Jul 26;23:36413648. [28]. Strumwasser A, Chu E, Yeung L, et al. A novel CT volume index score correlates with outcomes in polytrauma patients with pulmonary contusion. J Surg Res. 2011 Oct; 170(2):280-5.

28. Taheri Akerdi A, Mousavi SM, Abdolrahimzadehfard H, et al.Flail chest: Definition and management protocols need to be modified. J Trauma Acute Care Surg. 2021 Aug 1;91(2):e50..

29. Wanek S, Mayberry JC. Blunt thoracic trauma: flail chest, pulmonary contusion, and blast injury. Crit Care Clin. 2004 Jan;20(1):71-81.

30. Beks RB, Peek J, de Jong MB, et al. Fixation of flail chest or multiple rib fractures:current evidence and how to proceed. A systematic review and meta-analysis. Eur J Trauma Emerg Surg. 2019 Aug;45(4):631644. 
31. Senekjian L, Nirula R. Rib Fracture Fixation: Indications and Outcomes. Crit Care Clin. 2017 Jan;33(1):153-165.

32. Raimondi N, Vial MR, Calleja J,et al. Evidence-based guidelines for the use of tracheostomy in critically ill patients.J Crit Care. 2017 Apr;38:304-318.

33. Kang BH, Cho J, Lee JC, Jung K. Early versus late tracheostomy in trauma patients: a propensity-matched cohort study of 5 Years' data at a single institution in Korea. World J Surg. 2018; 42: 1742-174

\section{Table}

Table 3 is not available with this version.

\section{Figures}

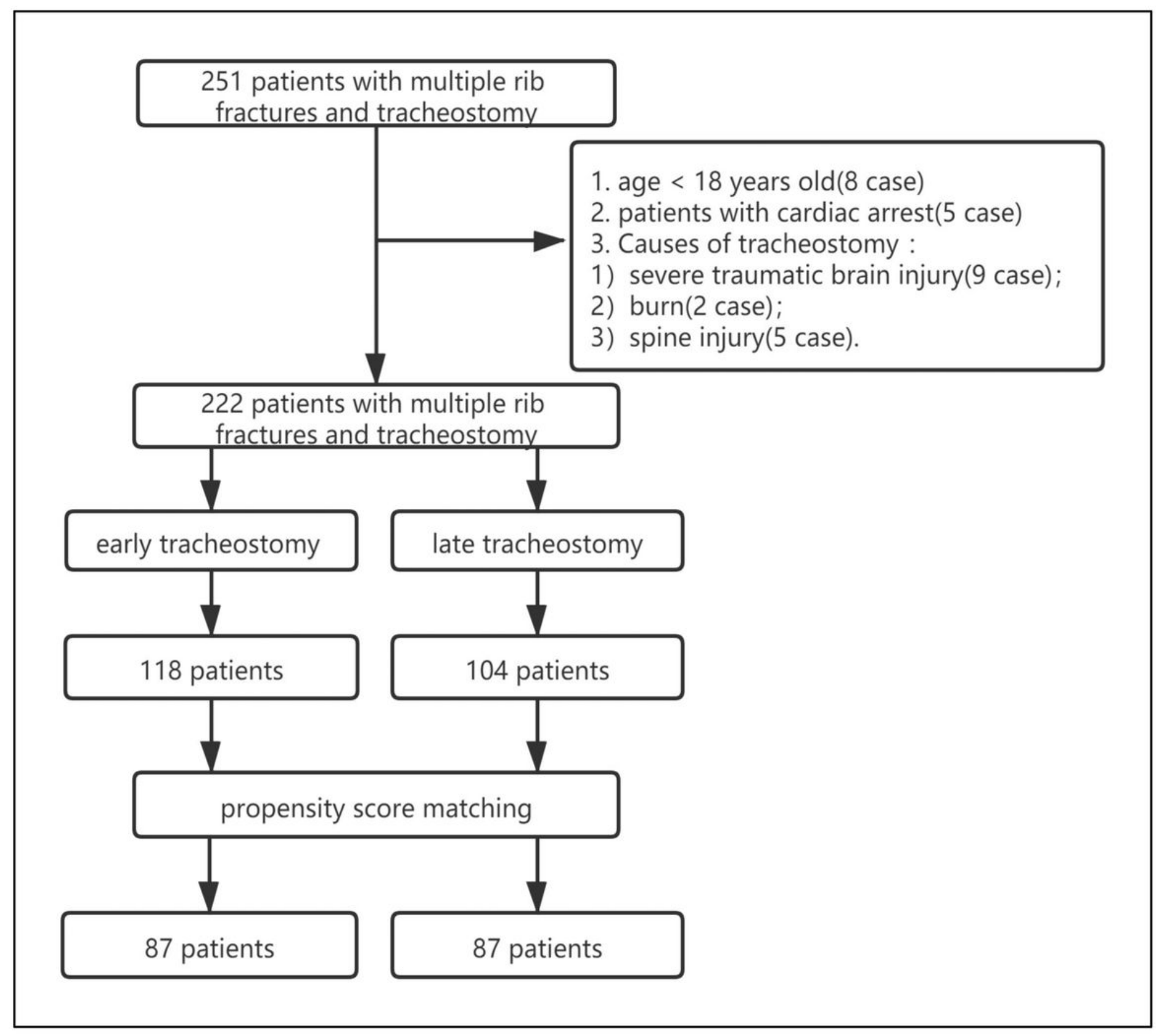


Figure 1

flow chart of study design and patient selection

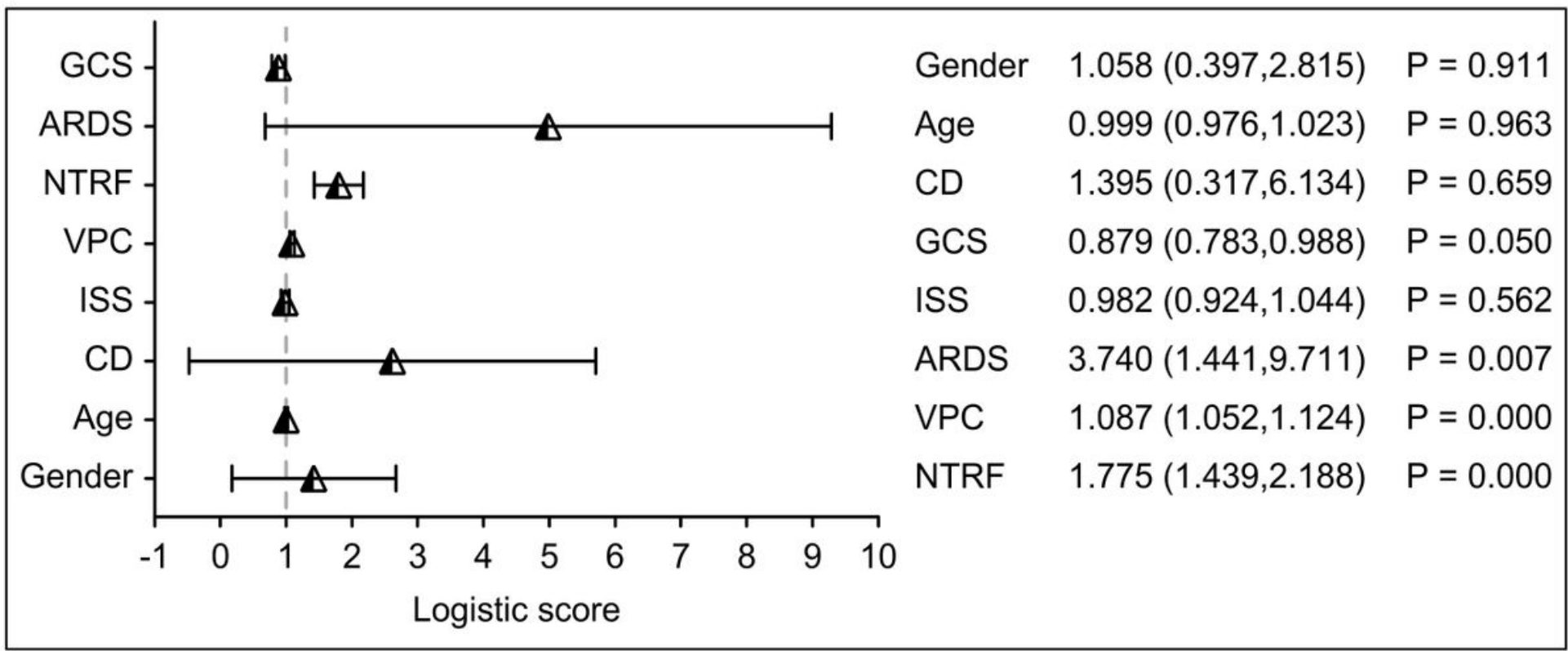

Figure 2

Results of multivariate logistic regression analysis of patients.

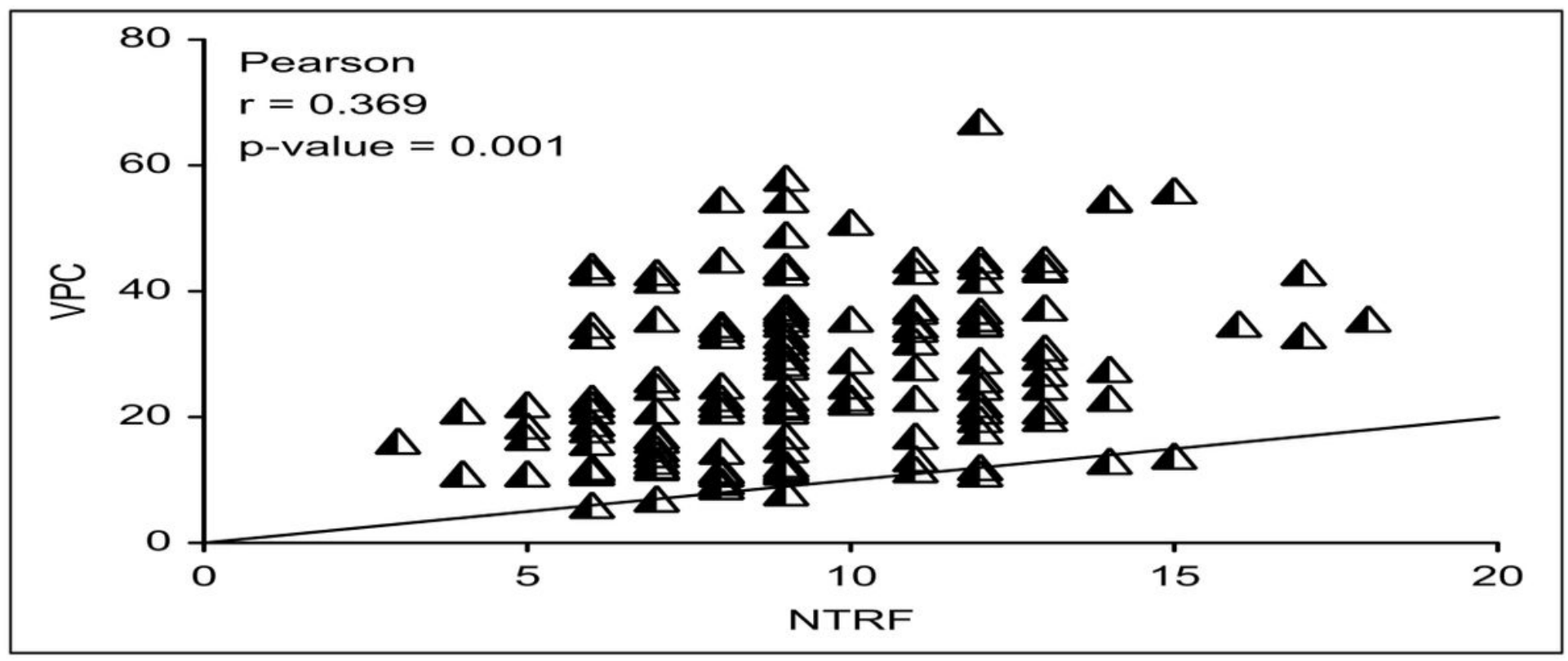

Figure 3

Correlation analysis results of volume of pulmonary contusion and total number of total fractures of the ribs. 
ROC Curve

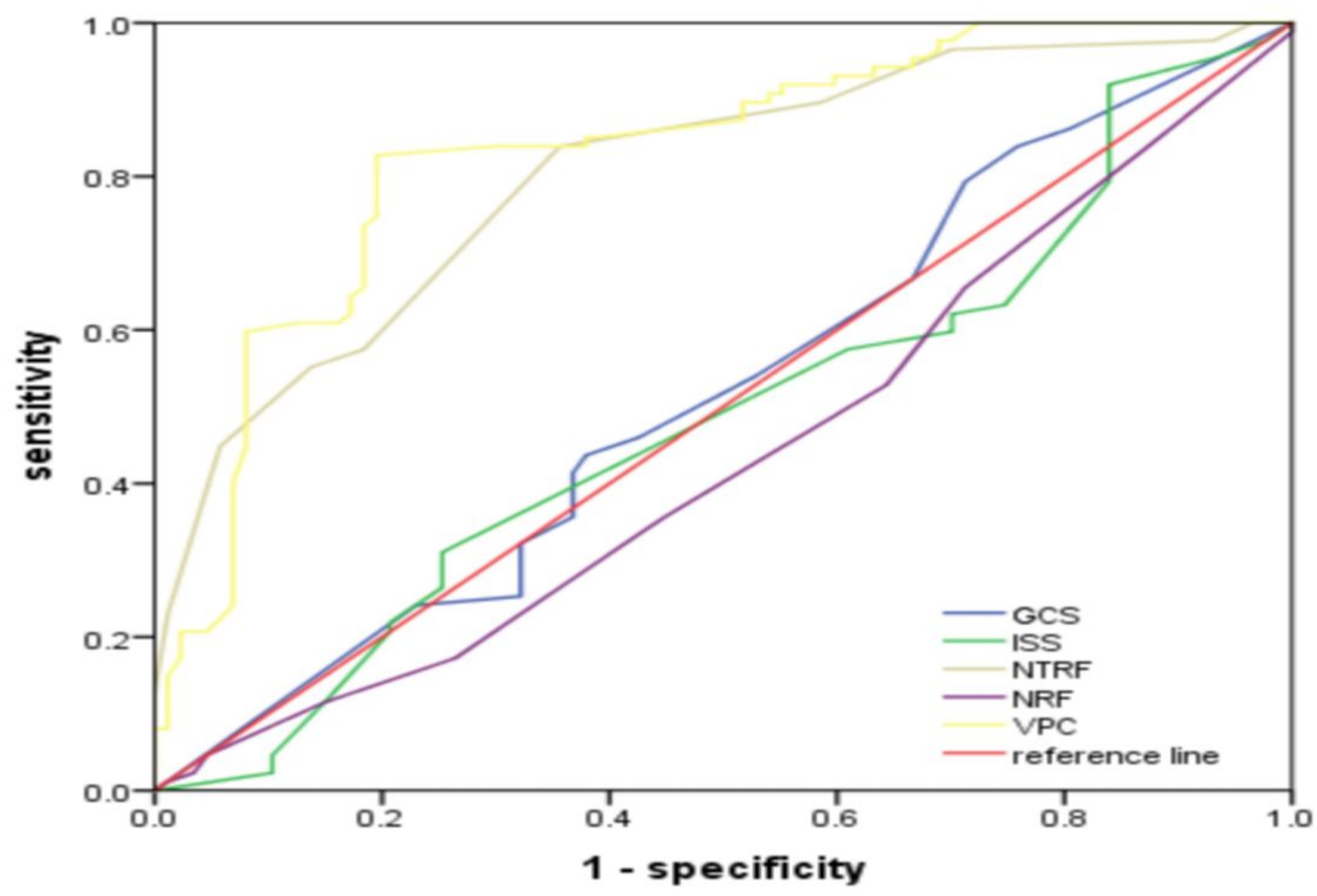

Figure 4

Results of ROC curve analysis. 


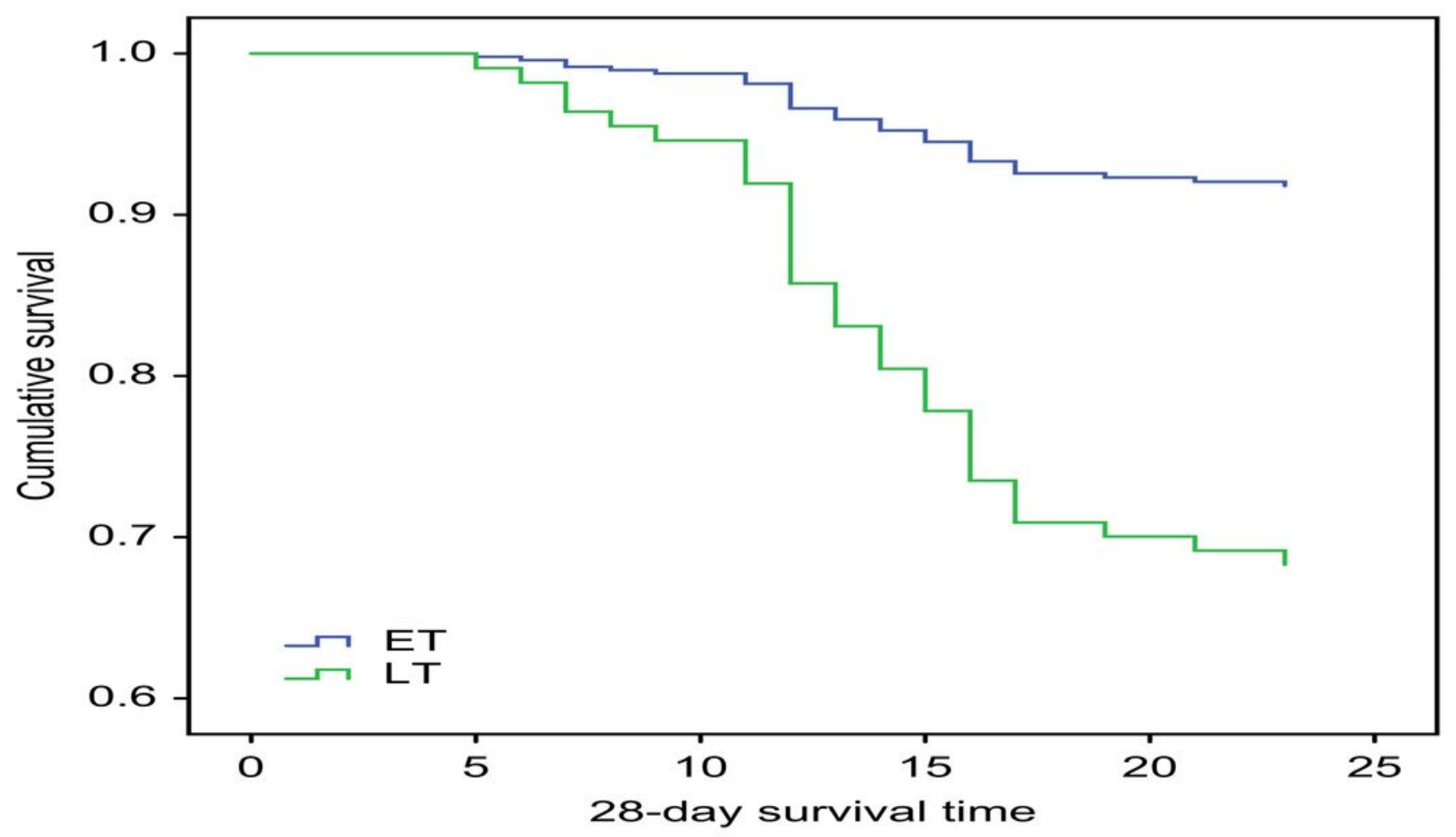

Figure 5

Kaplan-Meier survival analysis based on 28-day mortality. 